C2008 IEEE. Personal use of this material is permitted. However, permission to reprint/republish this material for advertising or promotional purposes or for creating new collective works for resale or redistribution to servers or lists, or to reuse any copyrighted component of this work in other works must be obtained from the IEEE. 


\title{
Optimal Scheduling of LTC and Shunt Capacitors in Large Distorted Distribution Systems Using Evolutionary-Based Algorithms
}

\author{
Agus Ulinuha, Mohammad A. S. Masoum, Senior Member, IEEE, and Syed M. Islam, Senior Member, IEEE
}

\begin{abstract}
The optimal scheduling of load tap changers (LTCs) and switched shunt capacitors for simultaneously minimizing energy loss and improving voltage profile while taking harmonics into account is performed using evolutionary-based algorithms (EAs). The proposed algorithm is capable of optimizing large distribution systems with different types of nonlinear loads. The decouple approach is employed for harmonic power-flow calculations, while two EAs are developed to determine the load interval division and near-optimal schedule. The inclusion of harmonics provides the optimization benefits while maintaining the acceptable distortion levels. The scheduling is carried out for the IEEE 123-bus with 14 shunt capacitors and 12 nonlinear loads. The main contributions are inclusion of harmonics in the optimal scheduling problem and its application to large distribution systems with multiple nonlinear loads.
\end{abstract}

Index Terms-Evolutionary algorithms (EAs), harmonics, large system, load tap changer (LTC) and shunt capacitors, optimal scheduling.

\section{INTRODUCTION}

$\mathbf{W}$ ITH a constantly changing electricity load, the operation of a distribution system has become quite complicated. If not carefully managed, the load variations may result in either the electricity demand not being fully satisfied or the electricity supplied to the customer has poor quality. The operation planning in the distribution system is therefore necessary to satisfy the demands in both technically acceptable and economically optimal. This essentially consists of scheduling load tap changers (LTCs) and switched shunt capacitors to prevent voltage violations and power-loss escalation due to load variations. The objective of the planning is to keep the voltage within the preset limits under changing load conditions while minimizing power losses.

The extensive applications of harmonic-generating devices in distribution system necessitate the planning to be extended by taking harmonics into account. Disregarding harmonics may lead to unacceptable results due to increased distortion levels and additional power losses [1]. As the real distribution systems

Manuscript received November 7, 2006; revised March 6, 2007. This work was supported by SPMU-TPSDP Universitas Muhammadiyah Surakarta under Contract 022/SD-TK/SPMU-UMS/VI/04. Paper no. TPWRD-00689-2006.

A. Ulinuha is with the Department of Electrical Engineering, Universitas Muhammadiyah Surakarta, Pabelan 57102, Indonesia.

M. A. S. Masoum and S. M. Islam are with the Department of Electrical and Computer Engineering, Curtin University of Technology, Perth 6845, Australia (e-mail: m.masoum@curtin.edu.au).

Color versions of one or more of the figures in this paper are available online at http://ieeexplore.ieee.org.

Digital Object Identifier 10.1109/TPWRD.2007.911166 usually consist of a large number of buses with different types of nonlinear loads, the planning needs to be carried out for the large distribution system involving a number of harmonic-generating devices. However, considering harmonics will lead the planning to be very complicated, significantly influencing the optimization problem [2], [3] and may generate different results. It may also reduce the optimization benefits [4].

Optimizing the distribution system for power-loss reduction and voltage profile improvement by optimally allocating a number of shunt capacitors while taking harmonics into account has been performed [5]-[7]. However, this was carried out for single load conditions. This paper considers load variations over a 24-h period and performs optimal scheduling using LTC and shunt capacitor banks.

Optimal scheduling of LTC and shunt capacitors is a multiphase decision-making problem with discrete variables and a nonlinear objective function [8]. The value of the objective is determined from power-flow solutions given the settings of control variables. Furthermore, it is preferable to achieve the objective value in the least possible number of control steps. The relation between bus voltage and control variables, which is highly nonlinear, makes the problem quite complicated [9]. On the other hand, the fulfillment of switching constraints will further lead the computation to be very intense. Conventional dispatch planning algorithms disregard harmonics in order to avoid further complexities.

This paper proposes an effective technique for the optimal scheduling problem while taking harmonic distortions into account. Evolutionary-based algorithms (EAs) are proposed for load interval division and near-optimal scheduling, while a relatively fast and accurate decoupled harmonic load-flow algorithm is developed and used as the backbone of the optimization problem. The optimization is carried out for the IEEE 123-bus system with five types of nonlinear loads connected to 12 buses.

\section{PROBLEM Formulation}

\section{A. Harmonic Power-Flow Calculation}

For a harmonic power-flow calculation, a decouple approach is developed and employed as a backbone of the optimization algorithm. This is justified due to the acceptable accuracy of the proposed decoupled harmonic power flow (DHPF) and the fact that industrial distribution systems consist of a large number of linear and nonlinear loads that cause convergence and memory storage problems if the harmonic couplings are accurately considered.

At harmonic frequencies, the system is modeled as a combination of passive elements and harmonic current sources. The 
related admittance matrix is modified according to the harmonic frequency [10], [11]. The general model of linear load as resistance in parallel with a reactance is utilized [12]. Nonlinear loads are modeled as current sources that inject harmonic current into the system. The fundamental and the $h$ th harmonic current of the nonlinear load installed at bus $i$ with real power $P$ and reactive power $Q$ are modeled as

$$
\begin{aligned}
& I^{(1)}=\left[\left(P_{i}+j Q_{i}\right) / V^{(1)}\right]^{*} \\
& I^{(h)}=C(h) I^{(1)}
\end{aligned}
$$

where $C(h)$ is the ratio of the $h^{\text {th }}$ harmonic current to its fundamental. The harmonic voltages are computed by solving the following load-flow equation:

$$
Y^{(h)} V^{(h)}=I^{(h)} .
$$

The rms voltage at bus $i$ is defined as

$$
V_{\text {irms }}=\left(\sum_{h=1}^{H}\left|V_{i}^{(h)}\right|^{2}\right)^{1 / 2}
$$

and the related total harmonic distortion of voltage $\left(\mathrm{THD}_{\mathrm{vi}}\right)$ is

$$
\mathrm{THD}_{\mathrm{vi}}=\frac{\left(\sum_{\mathrm{h} \neq 1}^{\mathrm{H}}\left|\mathrm{V}_{\mathrm{i}}^{(\mathrm{h})}\right|^{2}\right)^{1 / 2}}{\left|\mathrm{~V}_{\mathrm{i}}^{(1)}\right|^{1 / 2}} \times 100 \%
$$

where $H$ is the highest harmonic order considered.

At the $h$ th harmonic frequency, power loss in the line section between bus $i$ and $i+1$ is

$$
P_{\mathrm{loss}(\mathrm{i}, \mathrm{i}+1)}^{h}=R_{i, i+1}\left(\left|V_{i, i+1}^{(h)}-V_{i}^{(h)}\right|\left|y_{i, i+1}^{(h)}\right|\right)^{2}
$$

and the total power loss, including losses at harmonic frequencies, for an $m$ bus system is

$$
P_{\text {loss }}=\sum_{h=1}^{H}\left(\sum_{i=1}^{m} P_{\text {loss }(\mathrm{i}, \mathrm{i}+1)}^{(h)}\right) \text {. }
$$

\section{B. Optimization Problem Formulation}

The objective function of the scheduling problem is the minimization of energy loss over a 24-h period

$$
\min \sum_{t=1}^{24} P_{\mathrm{loss}}\left(Q_{t}, T_{t}\right)^{*} \Delta t
$$

where $P_{\text {loss }}$ is total power loss at hour $t$ as a function of $Q_{t}$ and $T_{t}$ that are the status of shunt capacitors and LTC tap position, respectively. While $\Delta t$ is the time interval that is normally taken as $1 \mathrm{~h}$. This objective function is subjected to the following constraints.

- Voltage constraint

$$
V_{i \min } \leq V_{\text {irms }} \leq V_{i \max }
$$

where $V_{i \min }$ and $V_{i \max }$ are the respective minimum and maximum limits of the rms voltage at bus $i\left(V_{\text {irms }}\right)$.
- Total harmonic distortion of voltage $\left(\mathrm{THD}_{\mathrm{v}}\right)$

$$
\mathrm{THD}_{\mathrm{vi}} \leq \mathrm{THD}_{\mathrm{v}}^{\max }
$$

where $\mathrm{THD}_{\mathrm{vi}}$ and $\mathrm{THD}_{\mathrm{v}}^{\max }$ are the distortion at bus $i$ and the maximum distortion allowed, respectively.

- Maximum switching operation of LTC

$$
\sum_{t=1}^{24}\left|\mathrm{TAP}_{\mathrm{t}}-\mathrm{TAP}_{\mathrm{t}-1}\right| \leq \mathrm{K}_{\mathrm{T}}
$$

where $\mathrm{TAP}_{\mathrm{t}}$ and $K_{T}$ are the LTC tap position at hour $t$ and maximum LTC switching, respectively.

- Maximum switching operation of shunt capacitors

$$
\sum_{t=1}^{24}\left(C_{n t} \oplus C_{n t-1}\right) \leq K_{c} ; \quad n=1,2, \ldots, n c
$$

where $C_{n t}$ and $K_{C}$ are the status of capacitor $n$ at hour $t$ and maximum switching allowed, respectively, while $n c$ is the number of shunt capacitors.

\section{SOLUTION PROPOSED}

The interdependence between the bus voltage and capacitor setting makes the optimization problem very complicated. The switching effects of shunt capacitors on the secondary bus voltage may cause an LTC tap position change, which forces LTC to operate too frequently, resulting in the reduction of LTC lifetime and higher maintenance cost. Some simplifications to avoid this oscillation have been proposed, such as the independent operation of LTC and shunt capacitors to separately control secondary bus voltage and reactive power [13] and the determination of capacitors' switching status prior to LTC tap status [14]-[19]. Unfortunately, these simplifications severely reduce the optimization benefits. Problem dimension is another difficulty that leads the optimization to encounter "dimensional exploration disaster" [20]. The maximum switching limitation of control devices makes computation very intense, as this constraint can only be confirmed after evaluating all states over the study period [21]. Furthermore, taking the harmonics into account results in an additional computational burden as the calculation should be extended for harmonic frequencies.

The aforementioned difficulties highlight the necessity of solving the problem effectively without reducing the solution accuracy. The interdependence between capacitor switching and LTC tap movement emphasizes the simultaneous scheduling of these switched elements. The problem with a very large dimension may be effectively taken in hand by employing the most suitable technique. To effectively satisfy the maximum allowable number of LTC switchings, the daily load curve will be divided into several intervals [22]. The optimal intervals determination should be effectively carried out by the chosen technique. The selected technique should also be capable of providing the possibility of checking the switching constraints of any possible schedule prior to calculating it. These will greatly reduce the computational burden due to unnecessary calculations for the infeasible schedules.

According to the aforementioned considerations, EAs are therefore proposed to be employed for this optimization problem. In addition to its general features, the proposed algorithms are justified due mainly to their encoding ability that 


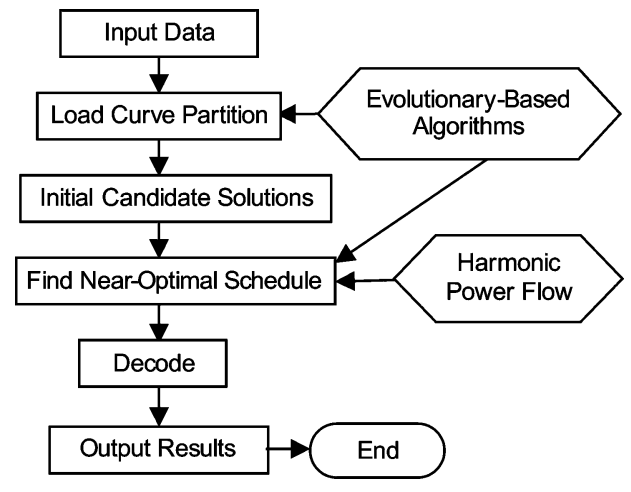

Fig. 1. Proposed flowchart for optimal scheduling of LTC and shunt capacitors while taking harmonics into account using EAs.

enables comprehensively considering the simultaneous scheduling of LTC and shunt capacitors and checking the switching constraints before performing unnecessary calculations.

\section{IMPLEMENTATION OF THE PROPOSED EAS}

EAs based on genetic concepts are robust and find near global optimal solutions as they perform a multidirectional search. They belong to the class of probability, but they are different from random algorithms as they combine elements of the directed search by maintaining the potential solutions. The disadvantage of this technique is the long processing time associated with it.

The proposed algorithms are initially constituted by a population of randomly generated candidate solutions, followed by a cycle of three stages: 1) evaluation of each candidate solution in the population; 2) selection of candidates for regeneration of new population; and 3) manipulation of candidates by crossover and mutation. Completing this cycle means that one iteration has occurred. After a number of iterations, the algorithm converges and the best candidate represents a near-optimal solution. In this paper, EAs are employed for the determination of a near-optimal load curve interval and a near-optimal dispatch schedule as shown in Fig. 1.

\section{A. Load Interval Division}

The idea of load interval division is based on the reality that several apparent load levels exist during a day. These intervals can therefore be used to determine the LTC tap position; which remains constant during a load interval and may differ at a different load interval. A typical load curve [23] is shown in Fig. 2 and will be used in this paper.

The load curve shown in Fig. 2 indicates that there are some intervals where the load variations are fairly small. If the load intervals can be properly determined, the schedule of the LTC tap position can be effectively established. This will not only take into account the overall daily load change, but also easily satisfy the switching constraint (11). By having the accurate result of load forecasting, the LTC dispatch obtained in this way can be practically implemented.

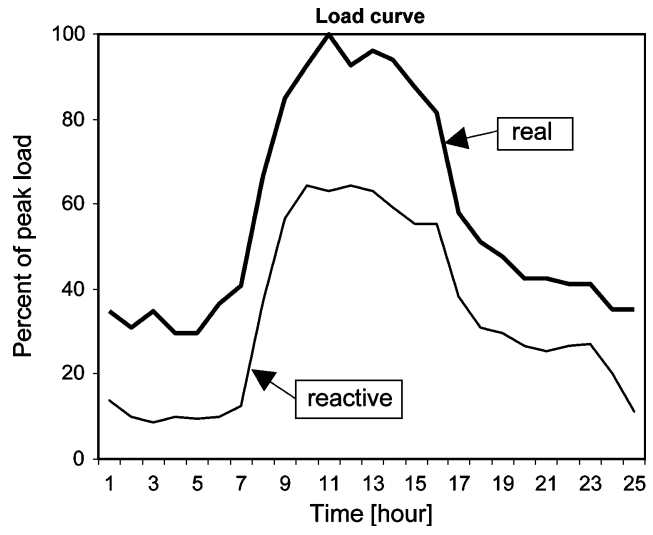

Fig. 2. Typical daily load curve.

TABLE I

Shunt CAPACITOR Data FOR THE IEEE 123-Bus System (Fig. 3)

\begin{tabular}{|c|c|c|}
\hline capacitor & location & kVAR \\
\hline C1 & 1 & 50 \\
C2 & 1 & 50 \\
C3 & 13 & 50 \\
C4 & 18 & 50 \\
C5 & 35 & 50 \\
C6 & 44 & 100 \\
C7 & 57 & 50 \\
C8 & 60 & 100 \\
C9 & 72 & 50 \\
C10 & 81 & 100 \\
C11 & 86 & 50 \\
C12 & 101 & 50 \\
C13 & 110 & 100 \\
C14 & 114 & 100 \\
\hline
\end{tabular}

In order to determine the intervals for the entire load curve that result in a near-optimal LTC schedule, the number of intervals is initially assumed. A single possible solution is called a candidate and consists of a number of binary substrings arranged in linear succession. An evolutionary-based algorithm is then employed to determine the intervals. For this purpose, the candidate that represents possible interval combination is constructed as

\begin{tabular}{cccccc}
\hline 0101 & 0011 & $\cdots$ & 0010 & $\leftarrow$ The candidate \\
\cline { 1 - 4 } 5 & 3 & $\cdots$ & 2 & $\leftarrow$ The substring value \\
$1^{\text {st }}$ & $2^{\text {nd }}$ & $\cdots$ & $n^{\text {th }}$ & $\leftarrow$ The $n$ intervals.
\end{tabular}

The candidate consists of a number of binary substrings according to the number of intervals considered. Each substring corresponds to the value indicating the length of an interval. The sum of all substring values indicates the total length of the entire interval, which is $24 \mathrm{~h}$. If the length of the substring is 4 , as the aforementioned case, the length of the candidate is $n \times 4$, where $n$ is the number of intervals assumed. A longer number of intervals may result in more precision of intervals; however, it will result in the longer candidate length, thereby increasing calculation cost. In this paper, the number of intervals considered is 4 and the length of the substring is 4 , so that the length 
of the candidate is 16. Candidates are evaluated using the following fitness function [22]:

$$
F=F_{\max }-\min \sum_{l=1}^{L} \sum_{t=1}^{T}\left[\left(P_{t l}-P A_{l}\right)^{2}+\left(Q_{t l}-Q A_{l}\right)^{2}\right] .
$$

Subject to

$$
\sum_{l=1}^{L} T=24
$$

where $F_{\max }$ is a constant that converts the fitness function to the standard form, $P_{t l}$ and $Q_{t l}$ are active and reactive powers (at hour $t$ and load interval $l$ ), $P A_{l}$ and $Q A_{l}$ are average active and reactive powers (at load interval $l$ ), $T$ is number of hour at the $l$ th load interval, and $L$ is the number of intervals assumed.

\section{B. Dispatch of LTC and Shunt Capacitors}

With the near-optimal load intervals in hand, the possible LTC tap position at every hour in a day can be determined. The construction of candidate for LTC tap scheduling is

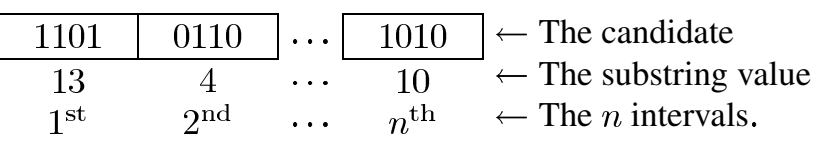

The candidate consists of a number of binary substrings according to the number of load intervals. In this paper, it is assumed that the difference between the consecutive LTC tap positions is no greater than 15 . Hence, the substring of $4 \mathrm{~b}$ is used. The eligible candidate is therefore that which has the sum of a substring value that is no more than the maximum allowable LTC switching operation.

For shunt capacitors at the substation, their switching operation is limited by the maximum allowable switching. Therefore, the candidate that represents the switching schedule for these shunt capacitors consists of several substrings where each substring denotes the switching status for the shunt capacitor for 24-h period. If the $i$ th bit is 0 , the status of that shunt capacitor at hour $i$ is "off." Therefore, the length of every substring is $24 \mathrm{~b}$ and the length of candidate for $s c$ shunt capacitors is $24(s c)$ bits. The candidate is eligible if the switching number in every substring is no more than the maximum switching limit.

The shunt capacitors installed at distribution feeders are normally allowed to be switched "on" and "off" once a day [22]. Therefore, it requires determining the time for switching the capacitor "on," and the duration for keeping it "on." The substring that represents the schedule for every shunt capacitor can be formed where the first segment represents the switch on time and the remaining represents the "on" duration. Since the latest time to switch it on or the maximum "on" duration is 24 , a segment of $5 \mathrm{~b}$ is used and, therefore, the length of substring is 10 b. For the following example of a substring:

\begin{tabular}{c|c|c}
0110 & 1101 & $\leftarrow$ The substring \\
\cline { 1 - 3 } 4 & 13 & $\leftarrow$ The segment value \\
$1^{\text {st }}$ & $2^{\text {nd }}$ & $\leftarrow$ The segment.
\end{tabular}

TABLE II

NONLINEAR LOAD DATA FOR THE IEEE 123-BUS SYSTEM (FIG. 3)

\begin{tabular}{|c|l|c|c|}
\hline nonlinear bus & nonlinear load type (Table A1) & kW & kVAR \\
\hline 13 & six-pulse 2 & 24.7 & 17.6 \\
15 & six-pulse 1 & 31.5 & 19.6 \\
21 & six-pulse 3 & 38.3 & 26.4 \\
25 & six-pulse variable frequency drive & 35.4 & 17.9 \\
40 & PWM adjustable speed drive & 47.4 & 29.3 \\
54 & six-pulse 2 & 38.2 & 15.1 \\
64 & six-pulse 1 & 21.3 & 9.3 \\
74 & six-pulse 3 & 25.7 & 17.2 \\
78 & six-pulse variable frequency drive & 38.4 & 14.2 \\
100 & PWM adjustable speed drive & 24.7 & 18.4 \\
106 & six-pulse 1 & 19.2 & 9.4 \\
610 & six-pulse variable frequency drive & 38.5 & 19.5 \\
\hline
\end{tabular}

The associated actual schedule is

\section{0.}

The eligible substring is that which has a total segment value of no more than 24 . The qualified candidate can only be formed by the eligible substrings. The length of candidate for the $f c$ feeder shunt capacitors is $f c \times 10$. Constructing the candidates in this way will greatly reduce its length, particularly for the system with a large number of feeder shunt capacitors.

The final eligible candidate representing the 24-h scheduling of LTC and shunt capacitor is consecutively constructed by the eligible candidate for LTC, substation shunt capacitors, and feeder shunt capacitors. For the optimization problem where the number of load intervals assumed is $n$, and the number of substation and feeder shunt capacitors is, respectively, $s c$ and $f c$, the length of candidate is $4(n)+24(s c)+10(f c)$.

Every candidate at each iteration is evaluated by the following fitness function:

$$
\begin{aligned}
F=\operatorname{Max}\left[F_{\max }-(w e E+\right. & w v \sum_{t=1}^{24} \sum_{i=1}^{m} \Delta V_{i t} \\
& \left.\left.+w t \sum_{t=1}^{24} \sum_{i=1}^{m} \Delta \mathrm{THD}_{\mathrm{it}}\right)\right]
\end{aligned}
$$

where $E$ is the per-unit energy loss, $\Delta V_{i t}$ is the per-unit rms voltage violation at bus $i$ at hour $t$, and $\Delta \mathrm{THD}_{\text {it }}$ is the perunit THD violation at bus $i$ at hour $t$ while $w e, w v$, and $w t$ denote the weighting coefficients of energy loss, rms voltage violation, and THD violation, respectively. The evaluation of every single candidate using the aforementioned fitness function requires running the decoupled harmonic power flow (DHPF) 24 times. This will increase the computing time.

\section{Simulation Results AND Discussion}

\section{A. System Data}

The IEEE 123-bus system [24] with the addition of 14 shunt capacitor banks (Table I) and 12 nonlinear loads (Table II), is used in this paper (Fig. 3). Nonlinear loads are modeled with 


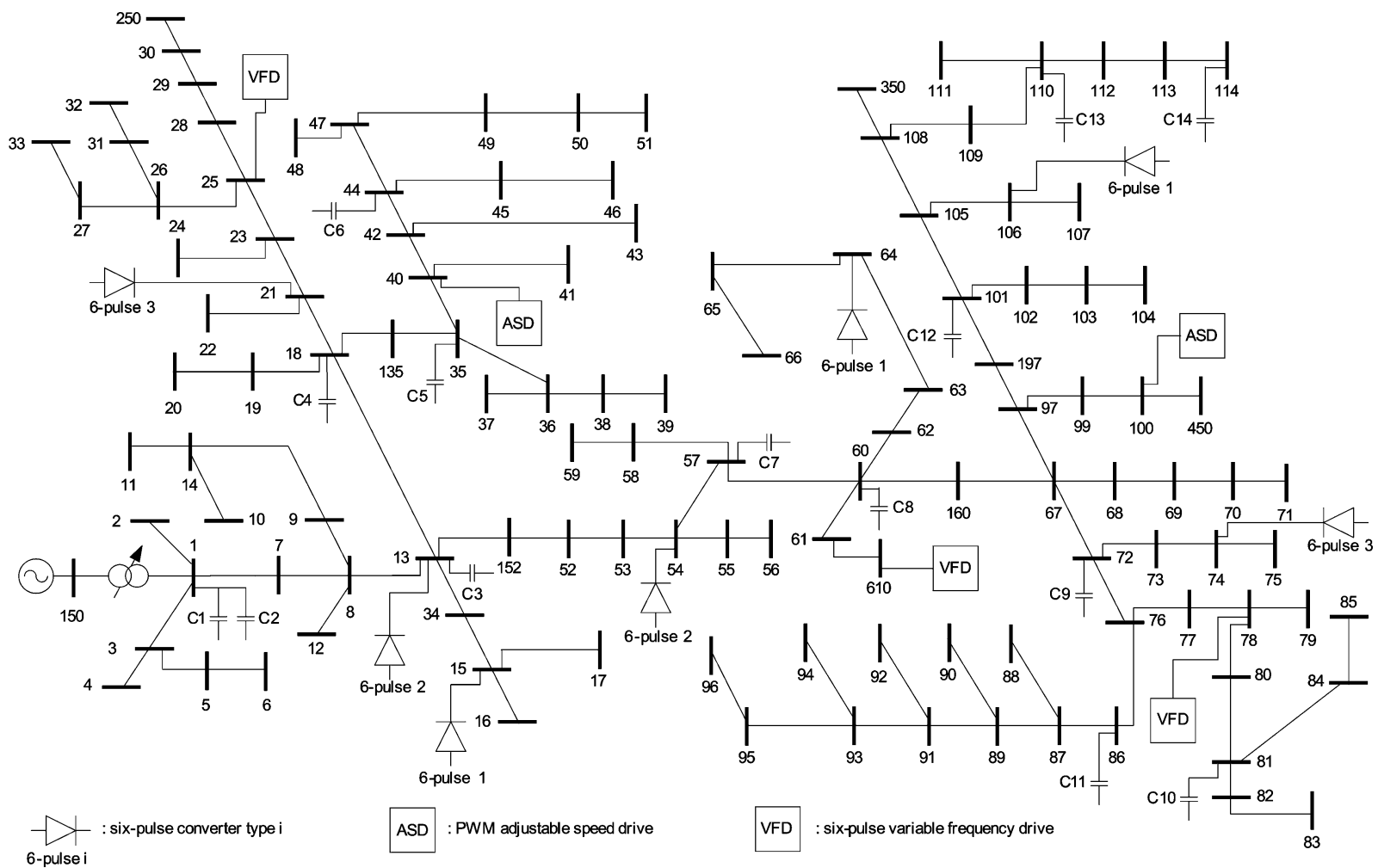

Fig. 3. IEEE 123-bus distorted distribution system used for simulations.

harmonic current sources (Table VI) and linear loads are presented as 50\% constant impedance and 50\% constant power. The peaks of both real and reactive loads are assumed to change according to the curve indicated in Fig. 2. The maximum and minimum voltage limits at every bus are 1.05 and 0.95 p.u., respectively. The allowable maximum switching operation of LTC is 30 , while the maximum switching allowed for shunt capacitors on substation and on the feeders is 6 and 2, respectively. Only the characteristic harmonics resulting from the nonlinear loads are considered to avoid complexity. Noncharacteristic harmonics may arise due to unbalance waveform and have not been considered in this work.

\section{B. Evolutionary Strategy of the Proposed Algorithms}

The initial candidates are randomly generated and those which satisfy the switching constraints are selected to construct the initial population. The crossover is carried out by selecting the candidates using the tournament method [25] and is performed by one-point crossover [26]. The probability of crossover and mutation, population size, and weighting coefficients are fixed throughout the iteration. The best candidate in every iteration is transferred directly to the next population. According to the selected number of load intervals and the system data

Length of candidate $=(4)(4)+(2)(24)+(12)(10)=184$.

Simulations are performed for 30 candidates and the number of iteration is set to 50 . The number of initial candidates as well as the number of iteration may be increased to achieve more accurate results; however, this will significantly increase the computation time. The crossover and mutation rates are set to
$60 \%$ and $1 \%$, respectively. Weighting coefficients influence the results and, on the other hand, different running of algorithms tends to generate slightly different results even for the same initial conditions. Finding the best compromised values for weighting coefficients, initial population, and iteration number are expected to generate accurate results with reasonable computation times. Simulations are performed using MATLAB version 7.0.1 R14 and a desktop PC (Pentium 4 Intel, 3.0-GHz processor and 512-MB RAM).

\section{Simulation Results for (Non) Sinusoidal Conditions}

The results of near-optimal scheduling of LTC and shunt capacitors for the IEEE 123-bus system under sinusoidal and nonsinusoidal (including the injected harmonics by the nonlinear loads) operating conditions are provided in Tables III and IV, respectively. The original tap position of the LTC is 0 and the initial status of all capacitors is "off" ( 0 ). Inspection of the near-optimal scheduling (Tables III and IV) indicates that all switching constraints are fully satisfied (Tables III and IV), voltage improvements are achieved (Fig. 4), and harmonic distortion is controlled (Fig. 6).

For both sinusoidal and nonsinusoidal operating conditions, bus 114 has the lowest voltage level during the optimization procedure (Fig. 4). The improved voltage levels are different for sinusoidal and nonsinusoidal conditions. The hourly reduction of real power losses achieved by the scheduling is shown in Fig. 5. It shows daily energy savings of $1024.00 \mathrm{kWh}$ and $1789.80 \mathrm{kWh}$ for optimization under sinusoidal and nonsinusoidal conditions, respectively.

One of the main objectives for inclusion of harmonics in the scheduling problem is to control the total harmonic distortion 
TABLE III

NEAR-OPTIMAL SCHEDULING OF LTC AND SHUNT CAPACITORS FOR THE IEEE 123-Bus System (Fig. 3) UNDER THE SinUSOIDAL OPERATING CONDITION

\begin{tabular}{|c|c|c|c|c|c|c|c|c|c|c|c|c|c|c|c|}
\hline \multirow[b]{2}{*}{$\stackrel{\Xi}{\Xi}$} & \multicolumn{15}{|c|}{ near-optimal schedule (sinusoidal operating conditions) } \\
\hline & U & $\bar{v}$ & U & రి & ठ & ש & ن & 已 & $\overbrace{}^{\infty}$ & 己 & 을 & $\bar{v}$ & ב & $\stackrel{m}{u}$ & $\bar{\nabla}$ \\
\hline 1 & 5 & 0 & 0 & 0 & 0 & 0 & 0 & 0 & 1 & 0 & 0 & 1 & 0 & 0 & 0 \\
\hline 2 & 5 & 0 & 0 & 0 & 0 & 0 & 0 & 0 & 1 & 0 & 0 & 1 & 0 & 0 & 0 \\
\hline 3 & 5 & 0 & 0 & 0 & 0 & 0 & 0 & 0 & 1 & 0 & 0 & 1 & 0 & 0 & 0 \\
\hline 4 & 5 & 0 & 1 & 0 & 0 & 0 & 0 & 0 & 1 & 0 & 0 & 1 & 0 & 0 & 0 \\
\hline 5 & 5 & 1 & 1 & 0 & 0 & 0 & 0 & 0 & 1 & 0 & 0 & 1 & 0 & 0 & 0 \\
\hline 6 & 5 & 1 & 1 & 1 & 0 & 0 & 0 & 0 & 1 & 0 & 0 & 1 & 0 & 0 & 0 \\
\hline 7 & 5 & 0 & 1 & 1 & 0 & 0 & 0 & 0 & 1 & 0 & 0 & 1 & 0 & 0 & 0 \\
\hline 8 & 3 & 0 & 1 & 1 & 0 & 0 & 0 & 0 & 1 & 1 & 0 & 1 & 0 & 1 & 0 \\
\hline 9 & 3 & 1 & 1 & 1 & 0 & 0 & 1 & 1 & 1 & 1 & 0 & 1 & 0 & 1 & 0 \\
\hline 10 & 6 & 1 & 1 & 1 & 0 & 1 & 1 & 1 & 1 & 1 & 1 & 1 & 0 & 1 & 0 \\
\hline 11 & 6 & 1 & 1 & 1 & 0 & 1 & 1 & 1 & 1 & 1 & 1 & 1 & 0 & 1 & 0 \\
\hline 12 & 6 & 1 & 1 & 1 & 0 & 1 & 1 & 1 & 1 & 1 & 1 & 1 & 0 & 0 & 1 \\
\hline 13 & 6 & 1 & 1 & 1 & 0 & 1 & 1 & & 1 & 1 & 1 & 1 & 0 & 0 & 1 \\
\hline 14 & 6 & 1 & 1 & 0 & 0 & 1 & 1 & 1 & 1 & 1 & 1 & 1 & 0 & 0 & 1 \\
\hline 15 & 6 & 0 & 1 & 0 & 0 & 1 & 1 & 1 & 1 & 0 & 1 & 1 & 0 & 0 & 1 \\
\hline 16 & 6 & 0 & 1 & 0 & 0 & 0 & 1 & 0 & 1 & 0 & 0 & 1 & 0 & 0 & 1 \\
\hline 17 & 3 & 1 & 1 & 0 & 0 & 0 & 1 & 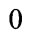 & 0 & 0 & 0 & 1 & 0 & 0 & 1 \\
\hline 18 & 3 & 1 & 1 & 0 & 1 & 0 & 1 & 0 & 0 & 0 & 0 & 1 & 0 & 0 & 1 \\
\hline 19 & 3 & 1 & 0 & 0 & 1 & 0 & 1 & 0 & 0 & 0 & 0 & 1 & 0 & 0 & 1 \\
\hline 20 & 3 & 1 & 0 & 0 & 0 & 0 & 1 & 0 & 0 & 0 & 0 & 1 & 0 & 0 & 1 \\
\hline 21 & 3 & 1 & 0 & 0 & 0 & 0 & 1 & 0 & 0 & 0 & 0 & 1 & 1 & 0 & 0 \\
\hline 22 & 3 & 1 & 0 & 0 & 0 & 0 & 1 & 0 & 0 & 0 & 0 & 1 & 0 & 0 & 0 \\
\hline 23 & 3 & 0 & 0 & 0 & 0 & 0 & 1 & 0 & 0 & 0 & 0 & 1 & 0 & 0 & 0 \\
\hline 24 & 3 & 0 & 1 & 0 & 0 & 0 & 0 & 0 & 0 & 0 & 0 & 0 & 0 & 0 & 0 \\
\hline
\end{tabular}

(THD) level of all buses and to maintain them within the recommended limits (e.g., IEEE 519 [27] and IEC 61000 [28]). Fig. 6 shows the THD reduction of the bus with the highest THD level (Fig. 3, bus 81), before and after the optimization. As expected, the proposed optimization algorithm has successfully limited all THD levels below the selected maximum value of 5\%.

The aforementioned results indicate the strong influence of harmonics on optimization results. The different generated schedules from the two different operating conditions result in different optimization benefits as indicated in Table V.

\section{Sinusoidal Near-Optimal Schedule Used for Nonsinusoidal Operation}

The impacts of harmonics are further highlighted using the sinusoidal schedule (Table III) for nonsinusoidal operating conditions. Simulation results indicate over voltage problems (e.g., at bus 150 (swing bus) from hour 10 to 15), lower energy savings of $1739.50 \mathrm{kWh}$, and unacceptable THD levels at some buses. This justifies the stipulation of including harmonics in the optimal dispatch scheduling of power systems in the present on nonlinear loads.

At hour 24, all buses have a THDv higher than the acceptable maximum level of 5\%. The lowest (5.53\%) and the highest
TABLE IV

NEAR-OPtimal SCHEdULING OF LTC AND SHUNT CAPACITORS FOR THE IEEE 123-BUS SYSTEM (FIG. 3) UNDER THE NONSINUSOIDAL OPERATING CONDITION

\begin{tabular}{|c|c|c|c|c|c|c|c|c|c|c|c|c|c|c|c|}
\hline \multirow[b]{2}{*}{$\stackrel{\Xi}{\Xi}$} & \multicolumn{15}{|c|}{ near-optimal schedule (nonsinusoidal operating conditions) } \\
\hline & $\underset{\sim}{\mathcal{U}}$ & $\bar{\tau}$ & U & & ठ & & e & & $\stackrel{\infty}{0}$ & U & 을 & $\overline{\bar{u}}$ & 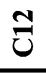 & $\frac{m}{v}$ & $\frac{\Delta}{U}$ \\
\hline 1 & 4 & 1 & 0 & 0 & 0 & 1 & 0 & 0 & 0 & 1 & 0 & 0 & 0 & 0 & 0 \\
\hline 2 & 4 & 1 & 0 & 0 & 0 & 1 & 0 & 0 & 0 & 1 & 1 & 0 & 0 & 0 & 0 \\
\hline 3 & 4 & 0 & 0 & 0 & 0 & 1 & 0 & 0 & 0 & 1 & 1 & 0 & 0 & 0 & 0 \\
\hline 4 & 4 & 1 & 0 & 0 & 0 & 1 & 0 & 0 & 0 & 1 & 1 & 0 & 0 & 0 & 0 \\
\hline 5 & 4 & 1 & 0 & 0 & 0 & 1 & 0 & 0 & 0 & 1 & 1 & 0 & 0 & 0 & 0 \\
\hline 6 & 4 & 0 & 0 & 1 & 0 & 1 & 0 & 0 & 0 & 1 & 1 & 0 & 0 & 0 & 0 \\
\hline 7 & -1 & 1 & 0 & 1 & 0 & 1 & 0 & 0 & 1 & 1 & 1 & 0 & 0 & 0 & 0 \\
\hline 8 & 5 & 1 & 0 & 1 & 0 & 1 & 1 & 0 & 1 & 1 & 1 & 0 & 0 & 0 & 0 \\
\hline 9 & 5 & 1 & 0 & 1 & 0 & 1 & 1 & 1 & 1 & 1 & 1 & 0 & 0 & 0 & 0 \\
\hline 10 & 5 & 1 & 1 & 1 & 1 & 1 & 1 & 1 & 1 & 1 & 1 & 0 & 1 & 0 & 0 \\
\hline 11 & 5 & 1 & 0 & 1 & 1 & 1 & 1 & 1 & 1 & 1 & 1 & 0 & 1 & 0 & 0 \\
\hline 12 & 5 & 1 & 0 & 1 & 1 & 1 & 1 & 1 & 1 & 1 & 1 & 1 & 1 & 1 & 0 \\
\hline 13 & 5 & 0 & 0 & 1 & 1 & 1 & 1 & 1 & 1 & 1 & 1 & 1 & 1 & 1 & 0 \\
\hline 14 & 5 & 0 & 0 & 1 & 1 & 1 & 1 & 1 & 1 & 1 & 1 & 1 & 1 & 1 & 0 \\
\hline 15 & 5 & 1 & 0 & 1 & 1 & 1 & 1 & 1 & 1 & 1 & 1 & 1 & 1 & 1 & 0 \\
\hline 16 & 5 & 1 & 1 & 1 & 1 & 0 & 1 & 1 & 1 & 1 & 1 & 1 & 1 & 1 & 0 \\
\hline 17 & 6 & 1 & 1 & 0 & 1 & 0 & 1 & 1 & 1 & 1 & 1 & 1 & 0 & 1 & 0 \\
\hline 18 & 6 & 1 & 1 & 0 & 1 & 0 & 1 & 1 & 1 & 1 & 0 & 0 & 0 & 1 & 0 \\
\hline 19 & 6 & 1 & 1 & 0 & 1 & 0 & 0 & 1 & 1 & 0 & 0 & 0 & 0 & 1 & 0 \\
\hline 20 & 6 & 1 & 1 & 0 & 1 & 0 & 0 & 1 & 1 & 0 & 0 & 0 & 0 & 1 & 0 \\
\hline 21 & 6 & 1 & 1 & 0 & 1 & 0 & 0 & 1 & 1 & 0 & 0 & 0 & 0 & 1 & 0 \\
\hline 22 & 6 & 1 & 1 & 0 & 1 & 0 & 0 & 0 & 1 & 0 & 0 & 0 & 0 & 1 & 0 \\
\hline 23 & 6 & 1 & 1 & 0 & 1 & 0 & 0 & 0 & 0 & 0 & 0 & 0 & 0 & 0 & 0 \\
\hline 24 & 6 & 1 & 1 & 0 & 0 & 0 & 0 & 0 & 0 & 0 & 0 & 0 & 0 & 0 & 0 \\
\hline
\end{tabular}

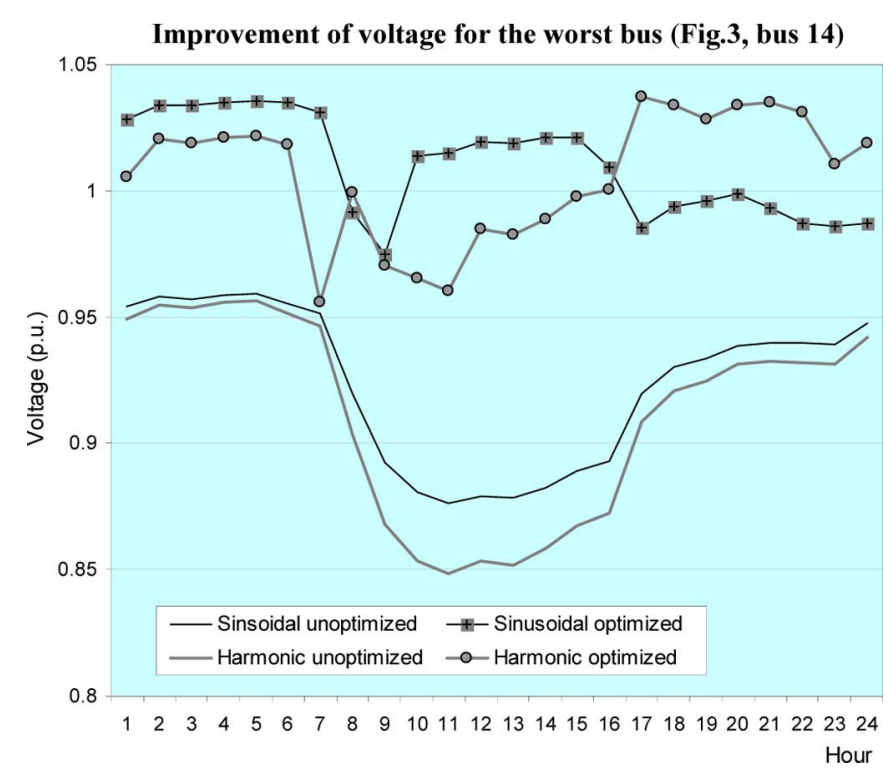

Fig. 4. Voltage improvement of the worst bus (Fig. 3, bus 114) under sinusoidal and nonsinusoidal (harmonic) operating conditions.

(6.46\%) THDv levels occur at buses 100 and 1, respectively. The lower energy savings are due to the fact that the voltage 


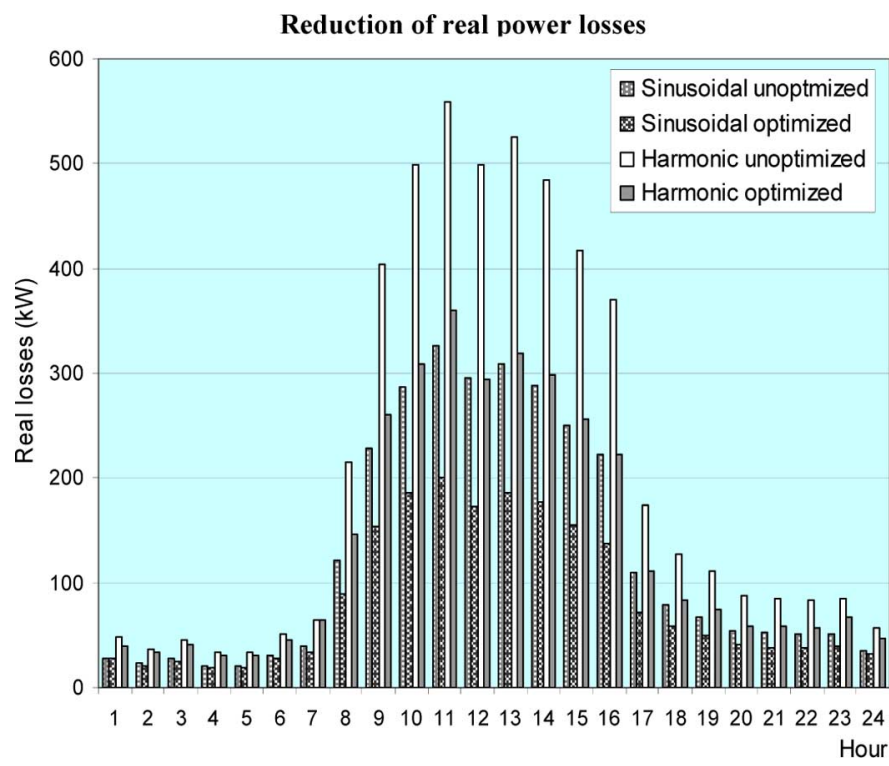

Fig. 5. Reduction of real power loss for the IEEE 123-bus system under sinusoidal and nonsinusoidal (harmonic) operating conditions.

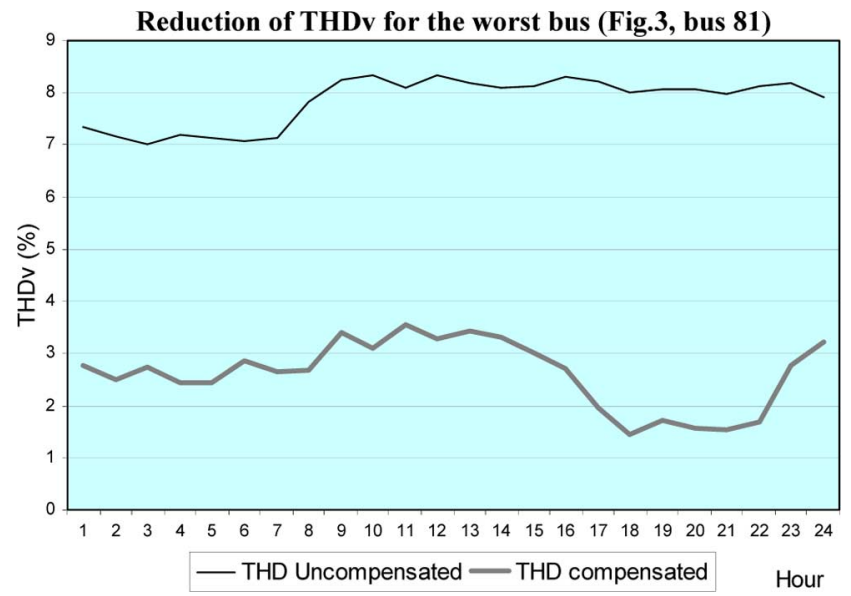

Fig. 6. Improvement (percentage of reduction) of voltage THD for the worst bus (Fig. 3, bus 81) under nonsinusoidal (harmonic) operating conditions.

TABLE V

BENEFITS AND COMPUTING TIMES OF NEAR-OPTIMAL DISPATCHING FOR (NON)SINUSOIDAL OPERATING CONDITIONS

\begin{tabular}{|l|l|l|}
\hline \multirow{2}{*}{ optimization benefits } & \multicolumn{2}{c|}{ operating conditions } \\
\cline { 2 - 3 } & \multicolumn{1}{|c|}{ sinusoidal } & \multicolumn{1}{c|}{ nonsinusoidal } \\
\hline average voltage improvement (\%) & 8.22 & 9.11 \\
total energy saving $(\mathrm{kWh})$ & 1024.00 & 1789.80 \\
computing time $(\mathrm{s}) *$ & 190.4 & 11590.2 \\
\hline
\end{tabular}

*) using Pentium 4 Intel 3.0-GHz processor

increments injected by harmonic currents have not been considered. Detailed inspection of the schedule under nonsinusoidal operating conditions (Table IV) denotes that capacitor C14 is never switched on during the scheduling period. This confirms that less compensation is needed for voltage improvement in harmonic scheduling optimization due to the harmonic voltage increment. However, the related average voltage improvement of the harmonic operating condition is higher than that of the sinusoidal operating condition as indicated in Table V. The result
TABLE VI

HARMONIC SPECTRUMS OF NONLINEAR LOADS (FIG. 3, TABLE II)

\begin{tabular}{|c|c|c|c|c|c|c|}
\hline \multirow{3}{*}{ order } & \multicolumn{2}{|c|}{ six-pulse 1 } & \multicolumn{2}{c|}{ six-pulse 2 } & \multicolumn{2}{c|}{ six-pulse 3 } \\
\cline { 2 - 7 } & $\begin{array}{c}\text { magnitude } \\
(\%)\end{array}$ & $\begin{array}{c}\text { phase } \\
\left({ }^{\circ}\right)\end{array}$ & $\begin{array}{c}\text { magnitude } \\
(\%)\end{array}$ & $\begin{array}{c}\text { phase } \\
\left({ }^{\circ}\right)\end{array}$ & $\begin{array}{c}\text { magnitude } \\
(\%)\end{array}$ & $\begin{array}{c}\text { phase } \\
\left({ }^{\circ}\right)\end{array}$ \\
\hline 1 & 100 & 0 & 100 & 0 & 100 & 0 \\
5 & 20 & 0 & 19.1 & 0 & 42 & 0 \\
7 & 14.3 & 0 & 13.1 & 0 & 14.3 & 0 \\
11 & 9.1 & 0 & 7.2 & 0 & 7.9 & 0 \\
13 & 7.7 & 0 & 5.6 & 0 & 3.2 & 0 \\
17 & 5.9 & 0 & 3.3 & 0 & 3.7 & 0 \\
19 & 5.3 & 0 & 2.4 & 0 & 2.3 & 0 \\
23 & 4.3 & 0 & 1.2 & 0 & 2.3 & 0 \\
25 & 4 & 0 & 0.8 & 0 & 1.4 & 0 \\
29 & 3.4 & 0 & 0.2 & 0 & 0 & 0 \\
31 & 3.2 & 0 & 0.2 & 0 & 0 & 0 \\
35 & 2.8 & 0 & 0.4 & 0 & 0 & 0 \\
37 & 2.7 & 0 & 0.5 & 0 & 0 & 0 \\
41 & 2.4 & 0 & 0.5 & 0 & 0 & 0 \\
43 & 2.3 & 0 & 0.5 & 0 & 0 & 0 \\
47 & 2.1 & 0 & 0.4 & 0 & 0 & 0 \\
49 & 2 & 0 & 0.4 & 0 & 0 & 0 \\
\hline
\end{tabular}

\begin{tabular}{|c|c|c|c|c|}
\hline \multirow{3}{*}{ order } & \multicolumn{2}{|c|}{ variable frequency drive } & \multicolumn{2}{c|}{ adjustable speed drive } \\
\cline { 2 - 5 } & $\begin{array}{c}\text { magnitude } \\
(\%)\end{array}$ & phase $\left(^{\circ}\right)$ & $\begin{array}{c}\text { magnitude } \\
(\%)\end{array}$ & phase $\left(^{\circ}\right)$ \\
\hline 1 & 100 & 0 & 100 & 0 \\
5 & 23.52 & 111 & 23.52 & 111 \\
7 & 6.08 & 109 & 6.08 & 109 \\
11 & 4.57 & -158 & 4.57 & -158 \\
13 & 4.2 & -178 & 4.2 & -178 \\
17 & 1.8 & -94 & 1.8 & -94 \\
19 & 1.37 & -92 & 1.37 & -92 \\
23 & 0.75 & -70 & 0.75 & -70 \\
25 & 0.56 & -70 & 0.56 & -70 \\
29 & 0.49 & -20 & 0.49 & -20 \\
31 & 0.54 & 7 & 0.54 & 7 \\
\hline
\end{tabular}

of running the sinusoidal schedule in the harmonic optimization environment also indicates lower energy savings of 1739.50 kWh.

\section{CONCLUSION}

Optimal scheduling of LTC and shunt capacitors for simultaneously reducing energy losses and improving the voltage profile while taking harmonics into account is performed using evolutionary algorithms based on genetic concepts. A decoupled harmonic power-flow algorithm is developed to include the effects of nonlinear loads and two algorithms are used for load interval division and device scheduling. Simulation results are presented for the IEEE 123-bus system with multiple nonlinear loads.

- The proposed evolutionary-based algorithms have effectively achieved the optimization goals under (non)sinusoidal operating conditions.

- The developed method also enables checking switching constraints prior to performing calculation for any possible schedule. This effectively reduces the computation burden due to unnecessary calculations for infeasible schedules.

- Harmonics have a great impact on the scheduling of LTC and shunt capacitors and including them in the optimization procedure is necessary to avoid unacceptable voltage violations, losses escalation, and THD levels.

- Inclusion of harmonics requires longer computing time. 
- Proper selection of optimization parameters (weighting functions, number of initial solutions, and iteration) will improve the solution and reduce the computing time.

- The application of the conventional optimal dispatch scheduling for nonsinusoidal operating conditions is not acceptable and will cause voltage limit violations, additional power losses, and high THD voltage distortions.

\section{APPENDIX}

Harmonic contents consisting of magnitude (\%) and angle (degree) of the five nonlinear loads employed in this paper are indicated in Table VI.

\section{REFERENCES}

[1] S. Ertem and Y. Baghzouz, "Optimal shunt capacitor sizing for distribution systems with multiple nonlinear loads," presented at the Industrial and Commercial Power Systems Tech. Conf., Detroit, MI, 1990.

[2] A. F. Zobaa, "A new approach for voltage harmonic distortion minimization," Elect. Power Syst. Res., vol. 70, no. 3, pp. 253-260, 2004.

[3] A. F. Zobaa and M. M. Aziz, "LC compensators based on transmission loss minimization for nonlinear loads," IEEE Trans. Power Del., vol. 19, no. 4, pp. 1740-1745, Oct. 2004.

[4] Z. Q. Wu and K. L. Lo, "Optimal choice of fixed and switched capacitors in radial distributors with distorted substation voltage," Proc. Inst. Elect. Eng., Gen., Transm. Distrib., vol. 142, no. 1, pp. 24-28, 1995.

[5] M. A. S. Masoum, M. Ladjevardi, A. Jafarian, and E. F. Fuchs, "Optimal placement, replacement and sizing of capacitor banks in distorted distribution networks by genetic algorithms," IEEE Trans. Power Del., vol. 19, no. 4, pp. 1794-1801, Oct. 2004.

[6] M. A. S. Masoum, A. Jafarian, M. Ladjevardi, E. F. Fuchs, and W. M. Grady, "Fuzzy approach for optimal placement and sizing of capacitor banks in the presence of harmonics," IEEE Trans. Power Del., vol. 19, no. 2, pp. 822-829, Apr. 2004.

[7] M. A. S. Masoum, M. Ladjevardi, E. F. Fuchs, and W. M. Grady, "Application of local variations and maximum sensitivities selection for optimal placement of shunt capacitor banks under nonsinusoidal operating conditions," Int. J. Elect. Power Energy Syst., vol. 26, no. 10, pp. 761-769, 2004.

[8] I. Roytelman, B. K. Wee, and R. L. Lugtu, "Volt/var control algorithm for modern distribution management system," IEEE Trans. Power Syst., vol. 10, no. 3, pp. 1454-1460, Aug. 1995.

[9] F.-C. Lu and Y.-Y. Hsu, "Fuzzy dynamic programming approach to reactive power/voltage control in a distribution substation," IEEE Trans. Power Syst., vol. 12, no. 2, pp. 681-688, May 1997.

[10] T. S. Chung and H. C. Leung, "A genetic algorithm approach in optimal capacitor selection with harmonic distortion considerations," Int. J. Elect. Power Energy Syst., vol. 21, no. 8, pp. 561-569, 1999.

[11] H.-C. Chin, "Optimal shunt capacitor allocation by fuzzy dynamic programming," Elect. Power Syst. Res., vol. 35, no. 2, pp. 133-139, 1995.

[12] Y. Baghzouz and S. Ertem, "Shunt capacitor sizing for radial distribution feeders with distorted substation voltage," IEEE Trans. Power Del., vol. 5, no. 2, pp. 650-657, Apr. 1990.

[13] R.-H. Liang and C.-K. Cheng, "Dispatch of main transformer ULTC and capacitors in a distribution system," IEEE Trans. Power Del., vol. 16, no. 4, pp. 625-630, Oct. 2001.

[14] G. Ramakrishna and N. D. Rao, "Fuzzy inference system to assist the operator in reactive power control in distribution systems," Proc, Inst. Elect. Eng., Gen., Transm. Distrib., vol. 145, no. 2, pp. 133-138, 1998.

[15] G. Ramakrishna and N. D. Rao, "Adaptive neuro-fuzzy inference system for volt/var control in distribution systems," Elect. Power Syst. Res., vol. 49, no. 2, pp. 87-97, 1999.

[16] G. Ramakrishna and N. D. Rao, "Implementation of a fuzzy logic scheme for Q/V control in distribution systems," presented at the IEEE Power Eng. Soc. Winter Meeting, 1999.
[17] J. J. Grainger and S. Civanlar, "Volt/var control on distribution systems with lateral branches using shunt capacitors and voltage regulators. Part I: The overall problem," IEEE Trans. Power App. Syst., vol. PAS-104, no. 11 , pp. $3278-3283$, Nov. 1985.

[18] J. J. Grainger and S. Civanlar, "Volt/var control on distribution systems with lateral branches using shunt capacitors and voltage regulators. Part III: The numerical result," IEEE Trans. Power App. Syst., vol. PAS-104, no. 11, pp. 3291-3297, Nov. 1985.

[19] J. J. Grainger and S. Civanlar, "Volt/var control on distribution systems with lateral branches using shunt capacitors and voltage regulators. Part II: The solution method," IEEE Trans. Power App. Syst., vol. 104, no. 11, pp. 3284-3290, Nov. 1985.

[20] Z. Gu and D. T. Rizy, "Neural networks for combined control of capacitor banks and voltage regulators in distribution systems," IEEE Trans. Power Del., vol. 11, no. 4, pp. 1921-1928, Oct. 1996.

[21] Y. Deng, X. Ren, C. Zhao, and D. Zhao, "A heuristic and algorithmic combined approach for reactive power optimization with time-varying load demand in distribution systems," IEEE Trans. Power Syst., vol. 17, no. 4, pp. 1068-1072, Nov. 2002.

[22] Z. Hu, X. Wang, H. Chen, and G. A. Taylor, "Volt/VAr control in distribution systems using a time-interval based approach," Proc. Inst. Elect. Eng., Gen., Transm. Distrib., vol. 150, no. 5, pp. 548-554, 2003.

[23] A. L. Shenkman, "Energy loss computation by using statistical techniques," IEEE Trans. Power Del., vol. 5, no. 1, pp. 254-258, Jan. 1990

[24] W. H. Kersting, "Radial distribution test feeders," IEEE Trans. Power Syst., vol. 6, no. 3, pp. 975-985, Aug. 1991.

[25] Z. Michalewics, Genetic Algorithms + Data Structures $=$ Evolution Program, 3rd ed. New York: Springer, 1996.

[26] D. T. Pham and D. Karaboga, Intelligent Optimization Technique: Genetic Algorithms, Tabu Search, Simulated Annealing and Neural Network. London, U.K.: Springer-Verlag, 1998.

[27] Recommended Practices and Requirements for Harmonic Control in Electrical Power Systems, IEEE, vol. Std.-519-1992, IEEE, 1993.

[28] Int. Electrotech. Comm.-IEC 61000 "Series: general guide on harmonics and interharmonics measurement and instrumentation," 2002.

Agus Ulinuha received the B.S. degree from Universitas Diponegoro, Semarang, Indonesia, in 1994, the M.S. degree from the Universitas Gadjah Mada, Indonesia, in 1999, and is currently pursuing the Ph.D. degree at Curtin University of Technology, Perth, Australia.

Currently, he is with the Academic Staff at the Department of Electrical Engineering, Universitas Muhammadiyah Surakarta, Indonesia. His research interest includes the optimization of electrical systems and harmonics.

Mohammad A. S. Masoum (SM'05) received the B.S., M.S., and Ph.D. degrees in electrical and computer engineering from the University of Colorado, Boulder, in 1983, 1985, and 1991, respectively.

He has published many papers in his area of expertise. His research interests include optimization, power quality and stability of power systems/electric machines, and distributed generation. Currently, he is an Associate Professor in the Electrical and Computer Engineering Department, Curtin University of Technology, Perth, Australia.

Syed M. Islam (SM'92) is the Professor of Electrical Power Engineering and Head of the Department of Electrical and Computer Engineering at the Curtin University of Technology, Perth, Australia. He has published many papers in his area of expertise. His research interests are in power systems, grid connection of renewable energy systems, power quality, and condition monitoring of transformers.

Prof. Islam was the recipient of the Dean's Medallion for Research in 1999 and the IEEE T Burke Haye's Faculty Recognition award in 2000. He is a Fellow of the Engineers Australia and a Fellow with the Institute of Electrical Engineers. 